\title{
Intrinsic Bioenergetic Properties and Stress Sensitivity of Dopaminergic Synaptosomes
}

\author{
Sung W. Choi, ${ }^{\star}$ Akos A. Gerencser, ${ }^{\star}$ Donna W. Lee, Subramanian Rajagopalan, David G. Nicholls, Julie K. Andersen, \\ and Martin D. Brand \\ Buck Institute for Research on Aging, Novato, California 94945
}

\begin{abstract}
Dopaminergic neurons of the substantia nigra pars compacta are defective in Parkinson's disease, but the specificity of this dysfunction is not understood. One hypothesis is that mitochondrial bioenergetic capacity is intrinsically lower in striatal dopaminergic presynaptic nerve varicosities, making them unusually susceptible to inhibition of electron transport by oxidative damage. To test this hypothesis, we separated isolated synaptosomes bearing dopamine transporters using immunomagnetic beads and compared their respiration with that of the residual nondopaminergic synaptosomes. As predicted, dopaminergic synaptosomes from striatum had lower respiratory rates. However, so did dopaminergic synaptosomes from cortex, indicating a lack of the predicted striatal specificity. We used fluorescent probes to analyze the bioenergetic competence of individual synaptosomes in the two fractions. The respiratory differences became nonsignificant when respiration rates were normalized to the number of respiration-competent synaptosomes, suggesting that differences reflected the quality of the different fractions. To circumvent damage induced by synaptosomal separation, we monitored membrane potentials in whole unseparated single synaptosomes using fluorescent imaging, and then identified the dopaminergic subpopulation using a fluorescent dopamine transporter substrate ( $\operatorname{ASP}^{+}[4$-(4-diethylaminostyryl)- $N$-methylpyridinium iodide]). The capacity of dopaminergic and nondopaminergic synaptosomes to maintain plasma membrane and mitochondrial membrane potential under several stresses did not differ. In addition, this capacity did not decline in either subpopulation with age, a risk factor for Parkinson's disease. We conclude that the intrinsic bioenergetic capacities of dopaminergic and nondopaminergic presynaptic synaptosomes from mice do not differ.
\end{abstract}

\section{Introduction}

Neuronal subtypes are differentially vulnerable to stress (Calabresi et al., 2000). One example is the preferential loss of dopaminergic neurons of the substantia nigra pars compacta in Parkinson's disease. Mitochondrial dysfunction is characteristic of the disease; complex I of the respiratory chain is less active in tissues from patients (Mizuno et al., 1989; Schapira et al., 1989; Shoffner et al., 1991) and in experimental models (Betarbet et al., 2000; Kumar et al., 2003; Chinta et al., 2007; Richardson et al., 2007). However, the selective vulnerability of dopaminergic neurons is not understood. They may be selectively exposed to oxidative stress, perhaps caused by reactive oxygen species generation during dopamine synthesis in the context of generally low oxidative defenses in neurons. This could lower complex I activity and cause chronic bioenergetic vulnerability. Indirect evidence supports this model, such as lower complex I activity and

Received Nov. 5, 2010; revised Jan. 15, 2011; accepted Jan. 31, 2011.

This work was supported by National Institutes of Health (NIH) Training Fellowship T32 AG000266 (S.W.C.) and NIH Grants P01 AG025901 (D.G.N., J.K.A., M.D.B.), NS041264 (J.K.A.), and P30 AG025708 (M.D.B.); the CHDI Foundation, Inc. (S.W.C., A.A.G., M.D.B.); The Larry L. Hillblom Foundation (D.W.L.); and The Keck Foundation (A.A.G., M.D.B., J.K.A., D.G.N.),

The authors declare no competing financial interests.

*S.W.C. and A.A.G. contributed equally to this work.

Correspondence should be addressed to Sung W. Choi, Buck Institute for Research on Aging, 8001 Redwood Boulevard, Novato, CA 94945. E-mail: swchoi.phd@gmail.com.

DOI:10.1523/JNEUROSCI.5817-10.2011

Copyright $\odot 2011$ the authors $\quad 0270-6474 / 11 / 314524-11 \$ 15.00 / 0$ less tolerance to complex I inhibition in dopaminergic neurons (Dawson and Dawson, 2003), and oxidative damage to cellular systems with ex vivo administration of dopamine (Rabinovic et al., 2000; Cantuti-Castelvetri et al., 2003; Miyazaki and Asanuma, 2008; Bisaglia et al., 2010). Dopamine also directly influences mitochondrial function; there are age-related alterations in mitochondrial morphology (Bertoni-Freddari et al., 1993, 1996, 2006), electron transport chain enzyme activity (Bowling et al., 1993; Ferrándiz et al., 1994; Guerrieri et al., 1996; Kilbride et al., 2008), and damage related to increased reactive oxygen species (Antier et al., 2004; Kilbride et al., 2008).

Aging is a major risk factor for Parkinson's disease. The nigrostriatum is particularly vulnerable to aging, but the relationships between aging, Parkinson's, and mitochondrial dysfunction remain unclear. One scenario is that bioenergetic capacity is limited in dopaminergic nerve terminals and synaptic varicosities, and decreases further with age until it fails to service peak energy demand, leading to bioenergetic collapse and death. Thus, bioenergetic dysfunction in dopaminergic neurons could play an early and critical role in Parkinson's (Bertoni-Freddari et al., 2006).

Synaptic terminals and varicosities are particularly relevant to neurodegenerative diseases associated with mitochondrial dysfunction, such as Parkinson's disease, since pathogenesis often starts at presynaptic regions (Betarbet et al., 2000). Because of high energy demand, presynaptic terminals are particularly sensitive to bioenergetic defects (Davey et al., 1997). Synaptosomes 
prepared from brain by homogenization and centrifugation are widely used to study mitochondrial bioenergetics and neurotransmitter synthesis and release. However, although the preparation avoids the requirement for neonatal tissue and in vitro culture that is inherent in primary cell culture, contamination with nonfunctional and non-presynaptic material and neurotransmitter heterogeneity have always been serious limitations of synaptosomal studies.

Here, we test whether dopaminergic synaptosomes have particularly low bioenergetic capacity that decreases with age and makes them selectively vulnerable to stress, using immunomagnetic separation of dopaminergic synaptosomes, respirometry, and single-synaptosome fluorescence microscopy. We introduce a fluorescent technique to identify individual dopaminergic synaptosomes in mixed fields of unpurified synaptosomes. We find no significant differences in bioenergetic capacity, or response to physiological challenges and aging, between dopaminergic and nondopaminergic synaptosomes.

\section{Materials and Methods}

Reagents. Tetramethylrhodamine methyl ester (TMRM), MitoTrackers, calcein-AM, and fura-4F AM were from Invitrogen. 4-(4-Diethylaminostyryl)$\mathrm{N}$-methylpyridinium iodide $\left(\mathrm{ASP}^{+}\right.$) and all other reagents were from Sigma-Aldrich, unless otherwise stated.

Animals. Young adult BL/6 mice of both genders, between 3 and 5 months of age, were used for experiments except where stated otherwise.

Synaptosomes. Nonfractionated synaptosomes used for single synaptosome imaging were prepared by Percoll gradient purification (Dunkley et al., 2008).

Dopaminergic synaptosomes were prepared as previously described (Chinta et al., 2007) with slight modifications. Briefly, the cortex from one mouse or striata from four mice were homogenized in $2 \mathrm{ml}$ of icecold solution A ( $320 \mathrm{~mm}$ sucrose, $1 \mathrm{~mm}$ K-EDTA, and $0.25 \mathrm{~mm}$ dithiothreitol, $\mathrm{pH}$ 7.4), and the homogenate was centrifuged at $1000 \times g$ for 10 $\min$ at $4^{\circ} \mathrm{C}$ to remove debris. The supernatant was incubated with antibody against the dopamine transporter (Alpha Diagnostic International; $25 \mu \mathrm{g} / \mathrm{sample}$ ) for $1 \mathrm{~h}$ at $4^{\circ} \mathrm{C}$. Then the mixture was centrifuged at $10,000 \times g$ for $2 \mathrm{~min}$, and the supernatant was removed. The pellet was resuspended in $1 \mathrm{ml}$ of solution $\mathrm{A}$ and recentrifuged. This resuspension and centrifugation step was repeated three times. The homogenized pellet was incubated with $150 \mu \mathrm{l}$ of secondary IgG magnetic beads (Miltenyi) for $45 \mathrm{~min}$ at $4^{\circ} \mathrm{C}$, and then poured into a magnetic column (MACS LS; Miltenyi) for separation of dopamine transporter- and magnetic bead-labeled dopaminergic synaptosomes (bound to column, DA) and the nondopaminergic fraction (flow through, FT).

Assay plates were coated before use with $0.0033 \%(\mathrm{v} / \mathrm{v})$ polyethyleneimine solution ( $100 \mu \mathrm{l} /$ well) overnight at room temperature, followed by Geltrex suspension (Invitrogen) diluted 1:100 with solution A (100 $\mu \mathrm{l} /$ well) for $1 \mathrm{~h}$ at $37^{\circ} \mathrm{C}$. Synaptosomes were attached by centrifugation to coated Seahorse V7 flux plates for respirometry at $20 \mu \mathrm{g}$ protein/well and to coverglass-bottomed 96-well microplates (Whatman) at $0.25 \mu \mathrm{g}$ of protein/well for microscopy (Choi et al., 2009). This was followed by dye loading for microscopic assays. Protein concentrations were determined by the Bradford assay (Bio-Rad).

Respirometry. The oxygen consumption rate of mitochondria within synaptosomes was determined in units of picomoles of $\mathrm{O}_{2} \cdot$ minute ${ }^{-1} \cdot 10$ $\mu \mathrm{g}$ of protein $^{-1}$ using a microplate-based respirometer (XF24; Seahorse Bioscience) as previously described (Gerencser et al., 2009). The assay buffer (S buffer) comprised $3.5 \mathrm{~mm} \mathrm{KCl,} 120 \mathrm{~mm} \mathrm{NaCl}, 1.3 \mathrm{~mm} \mathrm{CaCl}_{2}, 0.4$ $\mathrm{mm} \mathrm{KH}_{2} \mathrm{PO}_{4}, 1.2 \mathrm{~mm} \mathrm{Na}_{2} \mathrm{SO}_{4}, 15 \mathrm{~mm}$ D-glucose, $10 \mathrm{~mm}$ pyruvate, $0.4 \%$ (w/v) fatty acid-free bovine serum albumin, and $10 \mathrm{~mm}$ TES ( $N$ [tris(hydroxymethyl)methyl]-2-aminoethanesulfonic acid), pH 7.4. Nonmitochondrial respiration was defined as the average of three measurements after addition of $2 \mu \mathrm{M}$ rotenone plus $2 \mu \mathrm{M}$ myxothiazol (respiratory chain inhibitors), and this value for each well was subtracted from all other values for that well before calculation of the following respiration parameters: basal respiration (average value of the first three time points before any treatment); maximum respiration [first measurement value after addition of $4 \mu \mathrm{M}$ carbonylcyanide $p$-trifluoromethoxyphenylhydrazone (FCCP), an uncoupler of oxidative phosphorylation]; respiration driving proton leak (average value of three time points after addition of $4 \mu \mathrm{g} / \mathrm{ml}$ oligomycin, an inhibitor of ATP synthase); respiration driving ATP synthesis (basal respiration minus respiration driving proton leak); coupling efficiency $(100 \times$ respiration driving ATP synthesis/basal respiration); and spare respiratory capacity $(100 \times$ maximum respiration/basal respiration). The calculated values for each well were averaged for 3-10 technical replicate wells on one plate to give $n=1$ biological replicate.

Mitochondrial volume fraction in synaptosomes. The mitochondrial volume fraction was defined by confocal microscopic stereology as the volume density of mitochondria within synaptosomes and calculated as the total number of pixels corresponding to mitochondria (marked by MitoTracker Red) divided by the total number of pixels corresponding to the total synaptosomal volume (marked by calcein). Synaptosomes and their mitochondria lined up in the confocal optical plane. Because of the lack of random sectioning, the volume density was calculated as the $3 / 2$ nd power of the ratio of projected surfaces. This approach allowed counting of only mitochondrion-containing objects as synaptosomes. Synaptosomes were loaded with $2 \mu \mathrm{M}$ calcein-AM and $25 \mathrm{~nm}$ MitoTracker Red CMXRos for $45 \mathrm{~min}$ at $37^{\circ} \mathrm{C}$ and imaged in a Zeiss LSM 510 laser-scanning confocal microscope using a Plan-Apochromat $100 \times / 1.4$ oil lens. Single planes of $1024 \times 1024$ pixels were recorded at $44 \mathrm{~nm}$ pixel size at 1 Airy unit pinhole at high quality. Calcein and MitoTracker Red were simultaneously excited at $488 \mathrm{~nm}(30 \mathrm{~mW}$ Ar-ion laser at 10\% power) and at $543 \mathrm{~nm}(1.2 \mathrm{~mW} \mathrm{HeNe}$ laser at 100\%) and emissions were detected at $500-530 \mathrm{~nm}$ and above $560 \mathrm{~nm}$, respectively. These settings caused significant photodamage; therefore, each view field was scanned only once, and no $z$-sectioning was used. Each biological replicate was calculated from three view fields.

Recorded images were automatically analyzed in Image Analyst MKII (Image Analyst Software) using the VisualMacro feature. The diameter of fluorescent objects at their half-maximal intensity marks their real physical diameter despite the optical blurring (Gerencser et al., 2008). To binarize images by marking fluorescence objects at their half-maximal intensity above the local background, images were first high-pass filtered (Gerencser and Adam-Vizi, 2001) using a low-order (1.5) Butterworth filter at $\omega_{\text {cuton }}=0.67 \mu \mathrm{m} /$ cycle (Gerencser et al., 2008). As a result of this, the half-maxima of object intensities shifted to zero value. This was followed by smoothing using Wiener filtering, and rescaling to cutoff background and to provide an analysis independent of the brightness of the staining. Images were then segmented based on local maximum detection, watershed, and flood fill algorithms. Finally, only those calceinstained objects that overlapped with mitochondria were kept. For comparison of synaptosomal fractions, the same algorithm was used in an unbiased manner as no thresholds or parameters were manually set.

Quantification of respiration-competent synaptosomes. Synaptosomes were incubated with $50 \mathrm{~nm}$ MitoTracker Green (accumulated by membrane potential then covalently attached), 5 nм TMRM (reversibly accumulated by membrane potential), $1 \mu \mathrm{M}$ tetraphenylboron (TPB ${ }^{-}$) (aids TMRM uptake), and $2 \mu \mathrm{M}$ fura- $4 \mathrm{~F} \mathrm{AM}$ ( $\left[\mathrm{Ca}^{2+}\right]$ indicator) for $45 \mathrm{~min}$ at $37^{\circ} \mathrm{C}$ and imaged with a Nikon Eclipse Ti-PFS inverted epifluorescence microscope. To acquire $512 \times 512$ pixels images with a Cascade $512 \mathrm{~B}$ camera (Photometrics) at $0.267 \mu \mathrm{m} /$ pixel resolution using a S-Fluor $40 \times / 1.4$ oil lens, $1.5 \times$ optical zoom, a Lambda LB-LS17 Xe-arc light source (with partially attenuated intensity), 10-3 excitation and emission filter wheels (Sutter Instruments), and an MS-2000 linear encoded motorized stage (ASI) were used. The filter sets, given as excitationdichroic mirror-emission in nanometers/bandwidth, were as follows: for TMRM (at $50 \mathrm{~ms}$ exposure time): 543/22-562-617/73, for MitoTracker Green (300 ms) 472/30-495-520/35, and for fura-4F 340/26 (500 ms) and 387/11 (250 ms)-409-510/84 (all from Semrock). One central view field in each well was focused with the infrared laser-guided autofocusing system of the Nikon Eclipse Ti-PFS microscope, and typically 12 wells were imaged in a single experiment.

Recorded images were analyzed in Image Analyst MKII. First, all channels were subpixel aligned to MitoTracker Green fluorescence. Then the 
uneven background resulting from the wide-field configuration was removed by high-pass filtering at $\omega_{\text {cuton }}=0.037 \mu \mathrm{m} /$ cycle (Gerencser et al., 2008). Images were prepared for segmentation by Wiener filtering and rescaling, and the sum of the rescaled images was segmented as above. The segments were then used to evaluate their overlap with positive pixels in the binarized TMRM, MitoTracker Green, and fura-4F $340+$ $387 \mathrm{~nm}$ images. Objects were declared positive for a stain when at least $25 \%$ of the pixels overlapped with TMRM or MitoTracker Green or $50 \%$ of the fura-4F $340+387 \mathrm{~nm}$ images. Low $\left[\mathrm{Ca}^{2+}\right]$ objects were defined by the mean fura- $4 \mathrm{~F} 387 / 340$ reciprocal $\mathrm{Ca}^{2+}$ ratio (where high values denote low $\left[\mathrm{Ca}^{2+}\right]$ ) being above 1.5 , corresponding to $\sim 3 \mu \mathrm{M}\left[\mathrm{Ca}^{2+}\right]$. To normalize respiration rates to $10^{6}$ respiration-competent synaptosomes, rates were multiplied by $10^{6} \times(0.25 \mu \mathrm{g} / 10 \mu \mathrm{g}) \times(0.267 \times 512)^{2} /(\pi \times$ $\left.3200^{2}\right)=14.5$ and divided by the number of objects in a view field. In this formula, 0.25 and $10 \mu \mathrm{g}$ are the amounts of synaptosomes in the imaging and the Seahorse well, respectively; 0.267 is the resolution of imaging in micrometers/pixel, 512 is the image size in pixels, and 3200 is the radius of the imaging well in micrometers.

Wide-field imaging of $\mathrm{ASP}^{+}$fluorescence intensity. Striatal synaptosomes were incubated with $25 \mathrm{~nm}$ MitoTracker Red CMXRos in S buffer for $30 \mathrm{~min}$ at $37^{\circ} \mathrm{C}$ and wide-field epifluorescence imaged as above. One time point was acquired before addition of $\mathrm{ASP}^{+}(2 \mu \mathrm{M})$, and then five more images were taken at $90 \mathrm{~s}$ intervals by periodically visiting and autofocusing each well ( $\sim 20$ wells per experiment). The filter for MitoTracker Red (at $100 \mathrm{~ms}$ exposure time) was 582/15-593-610LP (the latter filter was from Omega Optical) and for $\mathrm{ASP}^{+}(200 \mathrm{~ms}$; unattenuated illumination) 472/30-495-542/27 (all from Semrock). The increase in $\mathrm{ASP}^{+}$fluorescence intensity in MitoTracker Red-positive structures was determined using Image Analyst MKII. Time series were backgroundsubtracted by high-pass filtering and aligned based on the MitoTracker Red image series to compensate for the incomplete registering of the $x, y$-motors during the time lapse. Then the MitoTracker Red time lapse was maximum intensity projected and binarized as detailed above. This binary mask was used to gate the fluorescence in the aligned $\mathrm{ASP}^{+}$image, to read out fluorescence intensity over synaptosomes, defined as mitochondrion-containing objects in the image.

Colocalization of $\mathrm{ASP}^{+}$fluorescence with immunofluorescence. Synaptosomes were incubated in the continuous presence $5 \mathrm{nM}$ TMRM and $1 \mu \mathrm{M}$ $\mathrm{TPB}^{-}$at $37^{\circ} \mathrm{C}$. After 40 min TMRM was wide-field epifluorescence imaged with attenuated light source as above. Then $\mathrm{ASP}^{+}(2 \mu \mathrm{M})$ was added and the same view fields were time lapse imaged for $10 \mathrm{~min}$ (five frames). After $\mathrm{ASP}^{+}$imaging, CellTracker Red CMTPX $(5 \mu \mathrm{M})$ was added to the synaptosomes, and after $5 \mathrm{~min}$ its fluorescence was acquired using the MitoTracker Red filter settings (see above; using 20 ms exposure time and unattenuated excitation). Then synaptosomes were fixed with $2 \%$ formaldehyde in Sorenson's phosphate buffer $\left(0.4 \mathrm{~mm} \mathrm{NaH}_{2} \mathrm{PO}_{4}, 160\right.$ $\mathrm{mM} \mathrm{Na}_{2} \mathrm{HPO}_{4}, \mathrm{pH} 7.3$ ) for $15 \mathrm{~min}$ and permeabilized with $0.1 \%(\mathrm{v} / \mathrm{v})$ Triton X-100 for $15 \mathrm{~min}$ at room temperature. Blocking was performed in $10 \%(\mathrm{v} / \mathrm{v})$ donkey serum (Jackson ImmunoResearch). Primary antibody against tyrosine hydroxylase (TH) (1:2000; AB152; Millipore) was applied overnight at $4^{\circ} \mathrm{C}$ followed by secondary anti-rabbit antibody conjugated with Alexa Fluor 488 (1:500; Invitrogen) for $30 \mathrm{~min}$ at room temperature. The microplate was replaced on the microscope, and coordinates stored during TMRM imaging were revisited. For increased accuracy, relative coordinates were used compared with a marker (grid) glued in an empty well. Alexa Fluor 488 (using filters described for MitoTracker Green) and CellTracker Red immunofluorescence were recorded and aligned with the live $\mathrm{ASP}^{+}$staining by matching CellTracker patterns. First, image backgrounds were subtracted by high-pass filtering and the live CellTracker images were aligned to the immunostaining by matching the live and the fixed CellTracker pattern. The TMRM and $\mathrm{TMRM}^{-\mathrm{ASP}^{+}}$time lapses were aligned in time and maximum intensity projected. Then both projections were aligned to match the live CellTracker image. All image alignments critical to the method were performed in Image Analyst MKII in a robust and automated manner using the principles of detection of two-dimensional cross-correlation maxima of the images using Fourier transformation. This was insensitive to the alteration of CellTracker pattern during immunolabeling or to the slight dissimilarity of TMRM and CellTracker patterns. For increased accuracy,
$6 \times$ oversampled images were used for calculation of the alignment. The $\mathrm{ASP}^{+}$and TH images were binarized as detailed above using SavitzkyGolay smoothing (Gerencser and Nicholls, 2008) before binarization. Finally, the functional TMRM image was segmented and the overlap of the segments with $\mathrm{ASP}^{+}$or with $\mathrm{TH}$ was evaluated, scoring overlaps $>25 \%$ as positive.

Wide-field imaging of membrane potential. Synaptosomes were isolated from the striata of young adult mice ( 5 months of age; mixed gender) or aged mice (28 months of age; mixed gender) and incubated in the presence of $5 \mathrm{nM}$ TMRM and $1 \mu \mathrm{M} \mathrm{TPB}^{-}$as above. Six time points were acquired at $5 \mathrm{~min}$ intervals, one view field per time point in each of the $\sim 24$ wells. Treatments were applied after acquisition of the first time point. At the end of the time lapse, samples were stained with $\mathrm{ASP}^{+}$and processed for TH immunofluorescence (see above). The functional TMRM fluorescence images were gated to show only fluorescence of those objects that initially accumulated TMRM and were stained or not stained either by $\mathrm{ASP}^{+}$or TH after the functional time lapse, using the technology detailed above. A mask was created by binarization of the temporal maximum intensity projection of the functional TMRM time lapse, and its intersection (i.e., objects with TMRM plus $\mathrm{ASP}^{+}$or $\mathrm{TH}$ fluorescence) and complement (objects with TMRM lacking $\mathrm{ASP}^{+}$or $\mathrm{TH}$ fluorescence) were calculated and used to monitor mean TMRM fluorescence of all dopaminergic and nondopaminergic objects, respectively, in the image. The change in TMRM fluorescence intensity reflects changes in both plasma membrane and mitochondrial membrane potentials, and was converted to millivolts relative to the baseline by the formula $26.7 \mathrm{mV} \times \ln \left(d F / F_{0}+1\right)$, where $d F$ is the change of TMRM fluorescence compared with its baseline $\left(F_{0}\right)$ (Gerencser and Nicholls, 2008). Membrane potential depolarization triggered by stressors was corrected for spontaneous drift measured in nontreated wells.

Statistics. Data are means \pm SE for $n$ biological replicates, each comprising 2-10 technical replicates. Statistical significance between groups (dopaminergic synaptosomes vs nondopaminergic synaptosomes) was tested by two-tailed, paired Student's $t$ test or ANOVA with Tukey's post hoc testing as appropriate.

\section{Results}

\section{Dopaminergic synaptosomes of the striatum prepared by immunomagnetic bead separation respire more slowly than nondopaminergic synaptosomes}

Synaptosomes prepared by conventional homogenization and centrifugation are a mixed population, making it difficult to distinguish the biological response of synaptosomes derived from one type of neuron from the response of the rest. Recent magnetic bead technology enables the separation of relatively pure dopaminergic synaptosomes from the flow-through residue containing other components of the synaptosomal suspension, mainly nondopaminergic synaptosomes and mitochondrial debris (Chinta et al., 2007).

Figure 1 shows that, when we compared oxygen consumption for the same quantity of protein $(10 \mu \mathrm{g})$, striatal dopaminergic synaptosomes had generally lower respiration rates under all conditions than the flow-through nondopaminergic fraction. Basal respiration (before addition of reagents), respiration driving ATP synthesis (prevented by addition of oligomycin to inhibit ATP synthase), respiration driving proton leak (not prevented by oligomycin), maximum respiration (after addition of uncoupler), and nonmitochondrial respiration (after addition of rotenone and myxothiazol to fully inhibit the mitochondrial electron transport chain) were all lower in dopaminergic synaptosomes than in the flow-through nondopaminergic fraction (Table 1). The lower respiration in dopaminergic synaptosomes was general and proportional, so the coupling efficiency (percentage of basal respiration used for ATP synthesis) and spare respiratory capacity (uncoupled respiration expressed as percentage of basal respiration) were not significantly different be- 


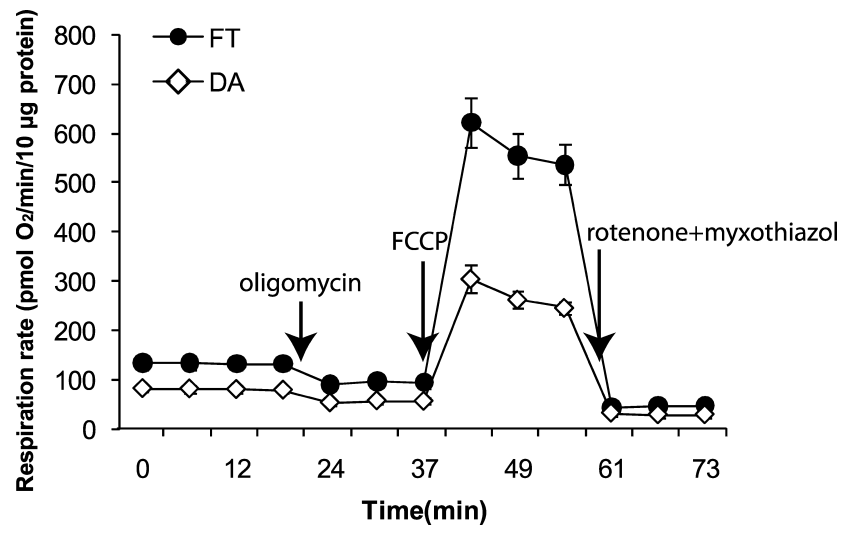

Figure 1. Isolated dopaminergic synaptosomes from striatum respire more slowly under all conditions than the flow-through fraction. Respiration of striatal dopaminergic synaptosomes (DA) (white diamonds) and flow-through nondopaminergic synaptosomes (FT) (black circles), after immunomagnetic bead separation. Glucose at $15 \mathrm{~mm}$ plus $10 \mathrm{~mm}$ pyruvate was used as substrate. Reagents were added as shown: oligomycin (4 $\mu \mathrm{g} / \mathrm{ml})$, FCCP $(4 \mu \mathrm{m})$, rotenone plus myxothiazol (each $2 \mu \mathrm{m})$. Data are means \pm SE for $n=5$ biological replicates, each the average of 3-10 technical replicates.

tween the two fractions (Table 1). These results appear to support the hypothesis that striatal dopaminergic synaptosomes have an intrinsically lower bioenergetic capacity than nondopaminergic synaptosomes from the same brain region.

\section{Slower respiration in dopaminergic synaptosomes was not striatum specific}

When dopaminergic synaptosomes were prepared from the cortex, respiration of dopaminergic synaptosomes was again lower than that of the flow-through fraction (Table 1). The respiration properties of cortical synaptosomes were not significantly different from the equivalent parameters for striatal synaptosomes under any condition. This result weakens the hypothesis that it is dopaminergic nerve terminals and varicosities in striatum that have a uniquely low bioenergetic capacity.

\section{Mitochondrial volume fraction in isolated dopaminergic and nondopaminergic synaptosomes is not different}

Lower respiration could be caused by a lower mitochondrial content and lower ATP demand in dopaminergic synaptosomes. Electron microscopy is typically used to measure the fraction of cellular volume occupied by mitochondria (the mitochondrial volume fraction), but it is difficult if not impossible to distinguish intact, respiration-competent synaptosomes from the large amounts of debris present in synaptosomal preparations (see below) using electron microscopy. As an alternative, we devised a confocal microscopic measurement of the mitochondrial volume fraction in live specimens, using two commonly used fluorescent probes, MitoTracker Red and calcein-AM (Fig. $2 A-C$ ). In live samples, these probes accumulate specifically in mitochondria and in all cellular compartments, respectively. The confocal optical plane thickness $(\sim 0.8 \mu \mathrm{m})$ allowed visualization of most of the spun-down synaptosomes and their mitochondria in a single plane. As only synaptosomes with mitochondria contribute to the measured respiration, we defined objects containing both probes (Fig. 2D), or in other words, in situ mitochondria enclosed by intact plasma membrane plus cytoplasm, as "intact synaptosomes."

The mitochondrial volume fraction within these intact synaptosomes did not differ significantly between dopaminergic synapto- somes and the flow-through fraction $\left(28 \pm 2 \%_{\mathrm{DA}}\right.$ vs $25 \pm 3 \%_{\mathrm{FT}}$ in striatum; $26 \pm 2 \%_{\mathrm{DA}}$ vs $26 \pm 1 \%_{\mathrm{FT}}$ in cortex) (Fig. $2 E$ ), ruling out the possibility that a smaller mitochondrial volume fraction caused lower respiration in dopaminergic synaptosomes. Our value of $\sim 26 \%$ is slightly lower than published electron microscopy results from others (Joyce et al., 2003). Interestingly, a significantly smaller fraction of calcein-accumulating objects contained mitochondria in the dopaminergic synaptosome preparation than in the flowthrough fraction (data not shown). This is analyzed by wide-field microscopy in detail below.

\section{Dopaminergic synaptosomal fractions contain fewer respiration-competent synaptosomes per microgram of protein than nondopaminergic fractions}

To refine the categorization of fluorescent objects shown in Figure 2, the assay described above was multiplexed by membrane potential and intracellular $\left[\mathrm{Ca}^{2+}\right]\left(\left[\mathrm{Ca}^{2+}\right]_{\mathrm{i}}\right)$ assays. The colocalization of three fluorescent probes, MitoTracker Green (green), fura-4F AM (blue), and TMRM (red), was analyzed by epifluorescence microscopy (Fig. $3 A-C, F, G$ ). Using image segmentation, we defined the following categories (Fig. $3 E, D, H$, Table 2).

\section{Type a: TMRM-positive}

Only intact synaptosomes containing polarized mitochondria and plasma membrane accumulate sufficient TMRM to be detected, so TMRM-positive objects were most probably respiring and were safely classified as "respiration-competent synaptosomes."

Type b: TMRM-negative, high-calcium

The TMRM-negative but fura-4F-positive objects were separated into two subgroups, high-calcium and low-calcium objects, based on the fura- $4 \mathrm{~F}$ signal. We used a fura- $4 \mathrm{~F}$ excitation ratio value equivalent to $3 \mu \mathrm{M}\left[\mathrm{Ca}^{2+}\right]_{\mathrm{i}}$ as the cutoff for "high-calcium"; this value is much higher than the physiological synaptosomal $[\mathrm{Ca}]_{\mathrm{i}}$ of $120 \mathrm{~nm}$ (Xiang et al., 1990). Any mitochondria inside such high-calcium objects were assumed to be nonfunctional and nonrespiring.

Type c: TMRM-negative, low-calcium, MitoTracker-positive Low-calcium objects that do not accumulate TMRM might have high glycolytic activity but no respiration, or have partially functional respiring mitochondria. MitoTracker Green accumulates into polarized mitochondria and then binds covalently. Mitochondria that were in synaptosomes and were polarized at some stage would accumulate MitoTracker Green and (unlike TMRM) retain it even if subsequently uncoupled and depolarized. MitoTracker Green-positive, TMRM-negative low-calcium objects were assumed to contain mitochondria that might be uncoupled but should still respire because calcium has not risen too high, so were also classified as respiration-competent synaptosomes.

\section{Type d: MitoTracker-positive only}

In our assay condition with $1.3 \mathrm{mM} \mathrm{CaCl}_{2}$, few free MitoTrackerpositive mitochondria were normally detected. However, MitoTracker-positive-only objects below the normal detection level were abundant at higher illumination intensity (data not shown). The fluorescence intensity of these objects was only a fraction of the fluorescence observed in mitochondria residing in synaptosomes. The low MitoTracker fluorescence intensity and the lack of fura-4F in all of these objects suggest that these free mitochondria underwent permeability transition pore opening in the presence of millimolar $\mathrm{Ca}^{2+}$, and therefore were incompetent at respiration. 
Table 1. Quantification of respiration rate parameters of immunomagnetically separated synaptosomal fractions

\begin{tabular}{|c|c|c|c|c|}
\hline & \multicolumn{2}{|l|}{ Striatum } & \multicolumn{2}{|l|}{ Cortex } \\
\hline & DA & FT & DA & FT \\
\hline Non-mitochondrial respiration $\left(\mathrm{pmol}_{2} \cdot \min ^{-1} \cdot 10 \mu \mathrm{g}\right.$ protein ${ }^{-1}$ ) & $27.7 \pm 4.3^{* *}$ & $44.5 \pm 3.3$ & $24.3 \pm 4.5^{*}$ & $37.8 \pm 5.6$ \\
\hline Basal respiration $\left(\mathrm{pmol}_{2} \cdot \min ^{-1} \cdot 10 \mu \mathrm{g}_{\text {protein }}{ }^{-1}\right)$ & $51.4 \pm 3.4^{*}$ & $88.9 \pm 10.6$ & $57.5 \pm 5.5^{* *}$ & $89.5 \pm 8.9$ \\
\hline Respiration driving ATP synthesis $\left(\mathrm{pmol}_{2} \cdot \min ^{-1} \cdot 10 \mu \mathrm{g}_{\text {protein }}{ }^{-1}\right.$ ) & $24.1 \pm 1.8^{* *}$ & $42.1 \pm 4.6$ & $23.4 \pm 2.6^{* *}$ & $35.8 \pm 3.1$ \\
\hline Respiration driving proton leak $\left(\mathrm{pmol}_{2} \cdot \min ^{-1} \cdot 10 \mu \mathrm{g}\right.$ protein $\left.^{-1}\right)$ & $27.4 \pm 2.9^{* *}$ & $51.9 \pm 5.5$ & $34.0 \pm 3.6^{* *}$ & $54.8 \pm 6.5$ \\
\hline Maximum respiration $\left(\mathrm{pmol}_{2} \cdot \min ^{-1} \cdot 10 \mu \mathrm{g}_{\text {protein }}{ }^{-1}\right.$ ) & $280 \pm 27^{* *}$ & $577 \pm 47$ & $301 \pm 23^{* *}$ & $562 \pm 38$ \\
\hline Coupling efficiency (\%) & $44.7 \pm 2.5$ & $43.6 \pm 3.2$ & $40.9 \pm 2.5$ & $37.3 \pm 4.7$ \\
\hline Spare respiratory capacity (\%) & $589 \pm 24$ & $648 \pm 40$ & $542 \pm 44$ & $625 \pm 21$ \\
\hline
\end{tabular}

Means \pm SE $[n=5$ biological replicates for striatum and $n=7$ biological replicates for cortex, each the average of $3-10$ technical replicates (wells) $]$ of respiratory parameters. The value for the non-mitrochondrial respiration rate in each technical replicate was subtracted from all other values. DA, Separated dopaminergic synaptosomal fraction; FT, residual flow-through fraction. Significance was tested by comparing the dopaminergic synaptosome value to the flow-through value from the same brain area, using two-tailed, paired Student's $t$ test.

${ }^{*} p<0.05,{ }^{* *} p<0.01$.
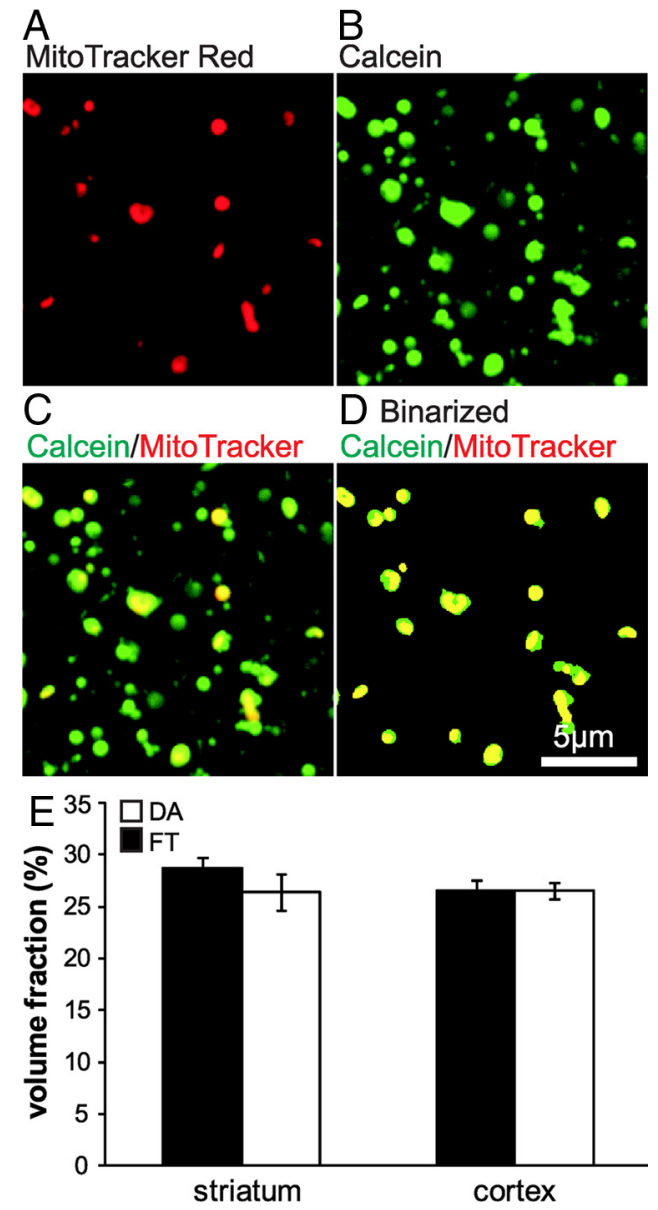

Figure 2. Volume fraction of mitochondria in different synaptosome populations. Representative confocal microscopic images of striatal immunomagnetic-purified dopaminergic synaptosomes. $\boldsymbol{A}$, Mitochondria marked by MitoTracker Red. $\boldsymbol{B}$, Cytosol marked by calcein. $\boldsymbol{C}$, Overlay of $\boldsymbol{A}$ and $\boldsymbol{B}$. D. Binarized image of $\boldsymbol{C}$ showing only mitochondrion-containing objects. $\boldsymbol{E}$, Mitochondrial volume fraction calculated as the $3 / 2$ nd power of the ratio given by dividing the number of yellow (mitochondria in red overlapping with cytosol in green) pixels by the number of green or yellow pixels in images similar to $\boldsymbol{D}$ for dopaminergic synaptosomes (DA) (white bars) or the flow-through fraction (FT) (black bars). Data are means $\pm \mathrm{SE}$ ( $n=6$ biological replicates each the average of 3 technical replicates).

Type e: low-calcium only

Low-calcium objects with no TMRM or MitoTracker signal were assumed to be glycolytic and nonrespiring.

We defined respiration-competent synaptosomes as those fluorescent objects that accumulated TMRM (type a), plus those that did not accumulate TMRM but nonetheless accumulated
MitoTracker Green while maintaining a relatively low cytosolic calcium concentration as reported by fura- $4 \mathrm{~F}$ (type c) (Fig. $3 I, J$ ).

The total number of objects detected by at least one of the three probes per microgram of protein was smaller in dopaminergic synaptosomes from both striatum and cortex (data not shown), presumably because of greater amounts of nonfunctional protein. In addition, a smaller proportion of fluorescent objects was identified as respiration-competent synaptosomes in the dopaminergic fraction (Fig. 3I). Therefore, the dopaminergic fraction had significantly fewer respiration-competent synaptosomes per microgram of protein than the flow-through fraction, and this was true in both cortex and striatum (Fig. $3 J$ ).

Respiration rates recalculated per $10^{6}$ respiration-competent synaptosomes were not different between dopaminergic and nondopaminergic fractions

To assess whether the lower respiration of dopaminergic synaptosomes seen in Figure 1 and Table 1 could be explained by the smaller number of competent synaptosomes per microgram of protein, the oxygen consumption rates were normalized to the number of competent synaptosomes defined as above. After this normalization, there were no significant differences in respiration rate under any condition between dopaminergic synaptosomes and the flow-through fractions derived from either striatum or cortex (Table 3 ). These results suggest that the differences in respiration rate between dopaminergic and nondopaminergic synaptosomes in Figure 1 and Table 1 were confounded by differences in the number of respiration-competent synaptosomes in different fractions.

\section{Identifying dopaminergic synaptosomes in a} mixed population

Immunomagnetic bead separation enabled the isolation of relatively pure dopaminergic synaptosomes, and therefore made possible a comparison of the respiration of dopaminergic and nondopaminergic synaptosomes. However, magnetic bead separation may cause differential damage to different synaptosomal fractions and allow different levels of contaminating nonfunctional protein. Therefore, to provide an alternative comparison of dopaminergic and nondopaminergic synaptosomes, we developed a method for identifying functional dopaminergic synaptosomes in mixed populations using a fluorescent marker. This enabled us to identify dopaminergic synaptosomes and then to compare their bioenergetic competence with nondopaminergic synaptosomes in the same field without physical separation. As a specific marker, we used $\mathrm{ASP}^{+}$, a fluorescent substrate of the monoamine transporter (Mason et al., 2005). The fluorescence intensity of $\mathrm{ASP}^{+}$was determined over MitoTracker Red-labeled 

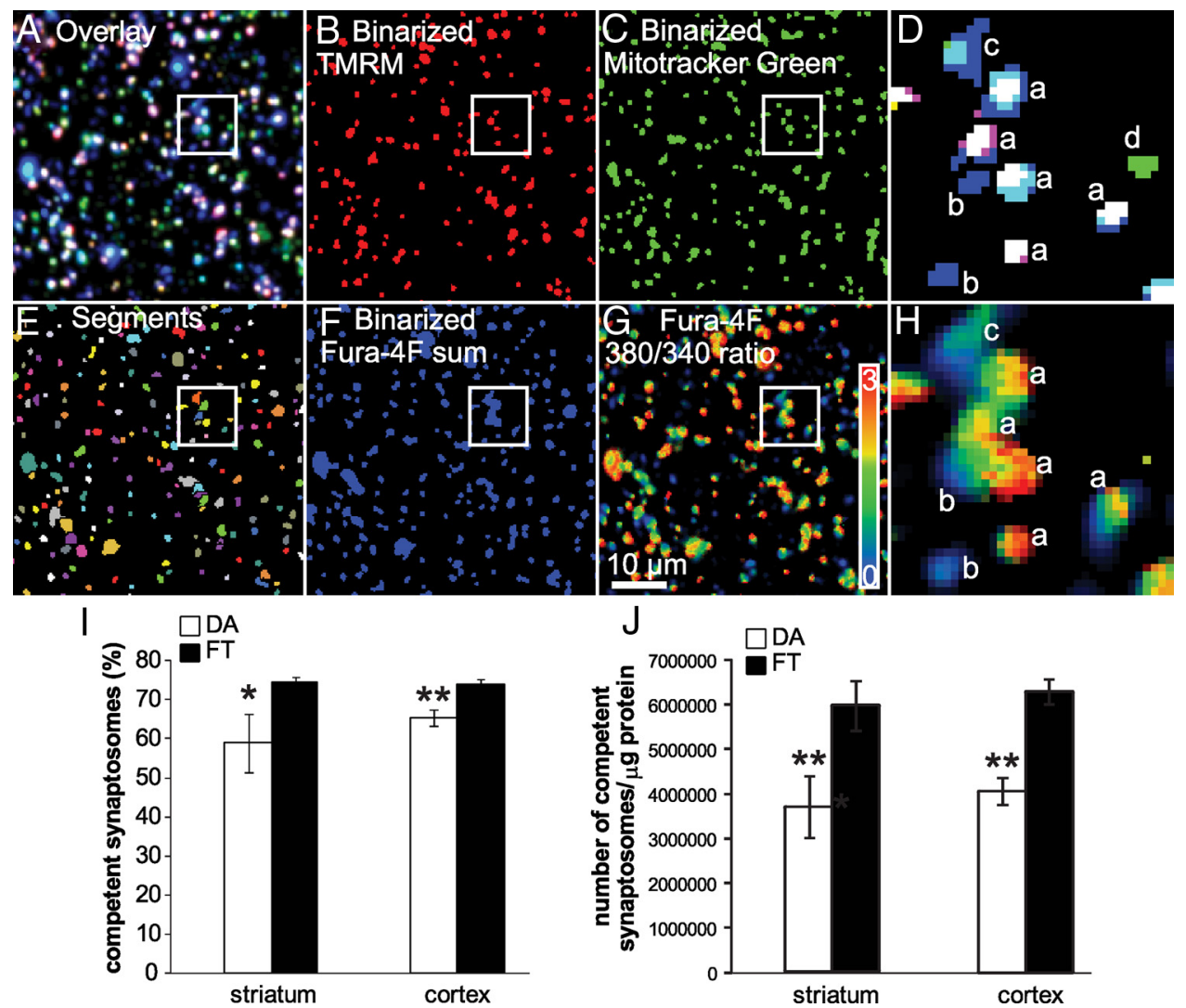

Figure 3. Quantification of bioenergetically competent synaptosomes. Synaptosomes prepared by immunomagnetic separation were probed with fura-4F AM ( $2 \mu \mathrm{m}$; blue), MitoTracker Green $(50 \mathrm{~nm}$; green), and TMRM ( $5 \mathrm{~nm}$; red). The three fluorescence channels are shown as a representative overlay image $(\boldsymbol{A})$, after binarization $(\boldsymbol{B}, \boldsymbol{C}, \boldsymbol{F})$, after reciprocal fura-4F ratio calculation $(\boldsymbol{G}, \boldsymbol{H})$, and after segmentation (E). The inset bar in $\boldsymbol{G}$ represents a color indicator of fura- $4 F$ ratio. $\boldsymbol{D}, \boldsymbol{H}$, Magnified overlay of images $\boldsymbol{B}, \boldsymbol{C}, \boldsymbol{F}$, and $\boldsymbol{G}$, respectively (indicated by rectangles) with the following objects marked: (a) TMRM-positive, (b) high-calcium, (c) low-calcium MitoTracker-positive, and (d) MitoTracker only. I, Percentage of the total number of objects per view field that are competent synaptosomes, defined as the synaptosomes that have (or once had) mitochondria with high membrane potential [categories (a) and (c) as defined in the text] in dopaminergic synaptosome preparations (DA) (white bars) and in the flow-through fractions (FT) (black bars). J, Numbers of bioenergetically competent synaptosomes per microgram of protein. Numbers were calculated as the sum of object counts defined in I multiplied by $\left(\pi \times 3200^{2}\right) /(0.267 \times 512)^{2} / 0.25 \mu \mathrm{g}$ (see Materials and Methods) to normalize to the total synaptosome content of a well. Data are means \pm SE of $n=5$ biological replicates, each the average of $2-10$ technical replicates. ${ }^{*} p<0.05$ or ${ }^{* *} p<0.01$ compared with FT from the same tissue.

Table 2. Classification of fluorescence objects in synaptosomal field-of-views

$\begin{array}{llll}\text { Striatum } & & \text { Cortex } & \\ & \text { DA } & \text { FT } & \text { FT }\end{array}$

Type a. TMRM-positive

Type b. TMRM-negative,

$51.0 \pm 8.1 \quad 66.6 \pm 1.4 \quad 57.0 \pm 0.4 \quad 66.2 \pm 1.1$ $29.8 \pm 6.2 \quad 16.6 \pm 1.9 \quad 25.3 \pm 2.9 \quad 17.9 \pm 1.3$

high-calcium

Type c. TMRM-negative, low-calcium, $7.9 \pm 1.2 \quad 7.9 \pm 0.4 \quad 8.5 \pm 1.5 \quad 7.7 \pm 0.3$ MitoTracker-positive

Type d. MitoTracker-positive only $\quad 6.3 \pm 0.9 \quad 7.0 \pm 1.3 \quad 6.2 \pm 1.1 \quad 6.4 \pm 0.5$

$\begin{array}{lllll}\text { Type e. Low-calcium only } & 4.9 \pm 1.9 & 1.9 \pm 0.4 & 2.9 \pm 0.6 & 10.7 \pm 0.3\end{array}$

Each subpopulation described in Fig. 3 was quantified. DA, Separated dopaminergic synaptosomal fraction; FT, residual flow-through fraction. Data are means $\pm \mathrm{SE}(n=5$ biological replicates, each the average of $2-3$ technical replicates).

structures using wide-field microscopy (Fig. 4A, G). The background subtraction technique (Gerencser and Adam-Vizi, 2001) allowed the $\mathrm{ASP}^{+}$associated with synaptosomes to be distinguished from the ASP ${ }^{+}$fluorescence in the bulk medium.

Synaptosomes accumulated $\mathrm{ASP}^{+}$in a time-dependent manner (Fig. $4 B-F)$. Accumulation was strongly inhibited by $50 \mathrm{~nm} 1-(2-$ (diphenylmethoxy)ethyl)-4-(3-phenylpropyl)piperazine (GBR12935) (Fig. 4H-L,M) or 10 nM 1-[2-[bis(4-fluorophenyl)methoxy]ethyl]4-(3-phenylpropyl)piperazine (GBR12909) (Fig. 4M). At these concentrations, GBR12935 and GBR12909 are selective inhibitors of the dopamine transporter while not inhibiting serotonin and norepi- nephrine transporters (Table 4). In contrast, $100 \mathrm{~nm}$ imipramine, which inhibits serotonin and norepinephrine transport but not dopamine transport (Table 4), had no effect on $\mathrm{ASP}^{+}$uptake by synaptosomes (Fig. 4M).

To confirm the specificity of $\mathrm{ASP}^{+}$tagging of dopaminergic synaptosomes in mixed synaptosome populations from striatum, we first stained synaptosomes for functional mitochondria with TMRM (Fig. 4N), and then determined the colocalization of the $\mathrm{ASP}^{+}$fluorescence signal (Fig. $4 S$ ) with a well established dopaminergic neuronal marker, tyrosine hydroxylase (TH) immunostaining (Fig. 4T). $\mathrm{ASP}^{+}$is not fixable; therefore, $\mathrm{ASP}^{+}$-exposed synaptosomes were stained with the fixable CellTracker Red (Fig. $4 O, P)$ and then processed for immunofluorescence staining. The microscope stage motorization and a robust subpixel precision image-registering algorithm allowed matching of images taken before and after histochemical processing by matching the CellTracker pattern (Fig. 4Q).

The TMRM images taken before $\mathrm{ASP}^{+}$addition were segmented (Fig. $4 R$ ), and the resultant objects were used to determine their overlaps with the probes (Fig. $4 U$ ). We found that $7 \pm 1$ and $28 \pm 2 \%$ of nonfractionated, TMRM-labeled striatal synaptosomes were positive for $\mathrm{ASP}^{+}$and TH, respectively (Fig. $4 U$, arrows) $(n=3)$. Overall, $88 \pm 4 \%$ of $\mathrm{ASP}^{+}$ objects colocalized with tyrosine hydroxylase, confirming the specificity of $\mathrm{ASP}^{+}$for dopaminergic synaptosomes. How- 


\begin{tabular}{|c|c|c|c|c|}
\hline & \multicolumn{2}{|l|}{ Striatum } & \multicolumn{2}{|l|}{ Cortex } \\
\hline & $\mathrm{DA}$ & FT & $\mathrm{DA}$ & FT \\
\hline Non-mitochondrial respiration $\left(\mathrm{pmol}_{2} \cdot \mathrm{min}^{-1} \cdot 10^{6}\right.$ synaptosomes ${ }^{-1}$ ) & $0.98 \pm 0.40$ & $0.90 \pm 0.22$ & $0.79 \pm 0.13$ & $0.76 \pm 0.05$ \\
\hline Basal respiration $\left(\mathrm{pmol}_{2} \cdot \mathrm{min}^{-1} \cdot 10^{6}\right.$ synaptosomes $\left.{ }^{-1}\right)$ & $1.65 \pm 0.41$ & $1.82 \pm 0.49$ & $1.52 \pm 0.18$ & $1.45 \pm 0.15$ \\
\hline Respiration driving ATP synthesis $\left(\mathrm{pmol}_{2} \cdot \mathrm{min}^{-1} \cdot 10^{6}\right.$ synaptosomes $^{-1}$ ) & $0.78 \pm 0.19$ & $0.84 \pm 0.20$ & $0.66 \pm 0.07$ & $0.59 \pm 0.05$ \\
\hline Respiration driving proton leak $\left(\mathrm{pmol}_{2} \cdot \mathrm{min}^{-1} \cdot 10^{6}\right.$ synaptosomes ${ }^{-1}$ ) & $0.88 \pm 0.22$ & $1.06 \pm 0.27$ & $0.87 \pm 0.12$ & $0.85 \pm 0.13$ \\
\hline Maximum respiration $\left(\mathrm{pmol}_{2} \cdot \mathrm{min}^{-1} \cdot 10^{6}\right.$ synaptosomes $^{-1}$ ) & $9.03 \pm 2.25$ & $11.61 \pm 2.68$ & $8.39 \pm 0.81$ & $9.31 \pm 0.73$ \\
\hline
\end{tabular}

Each respiration value in Table 1 was normalized by the number of bioenergetically competent synaptosomes per microgram of protein from the same experimental data set (Table 2 ) and expressed as pmol $0_{2} / \mathrm{min} / 10^{6}$ synaptosomes. DA Separated dopaminergic synaptosomal fraction; FT, residual flow-through fraction. Data are means $\pm \mathrm{SE}$ ( $n=5$ biological replicates for striatum and $n=7$ biological replicates for cortex, each the average of 3-10 technical replicates). Significance was tested by comparing the DA value with the FT value form the same tissue, no pairs had $p<0.05$.

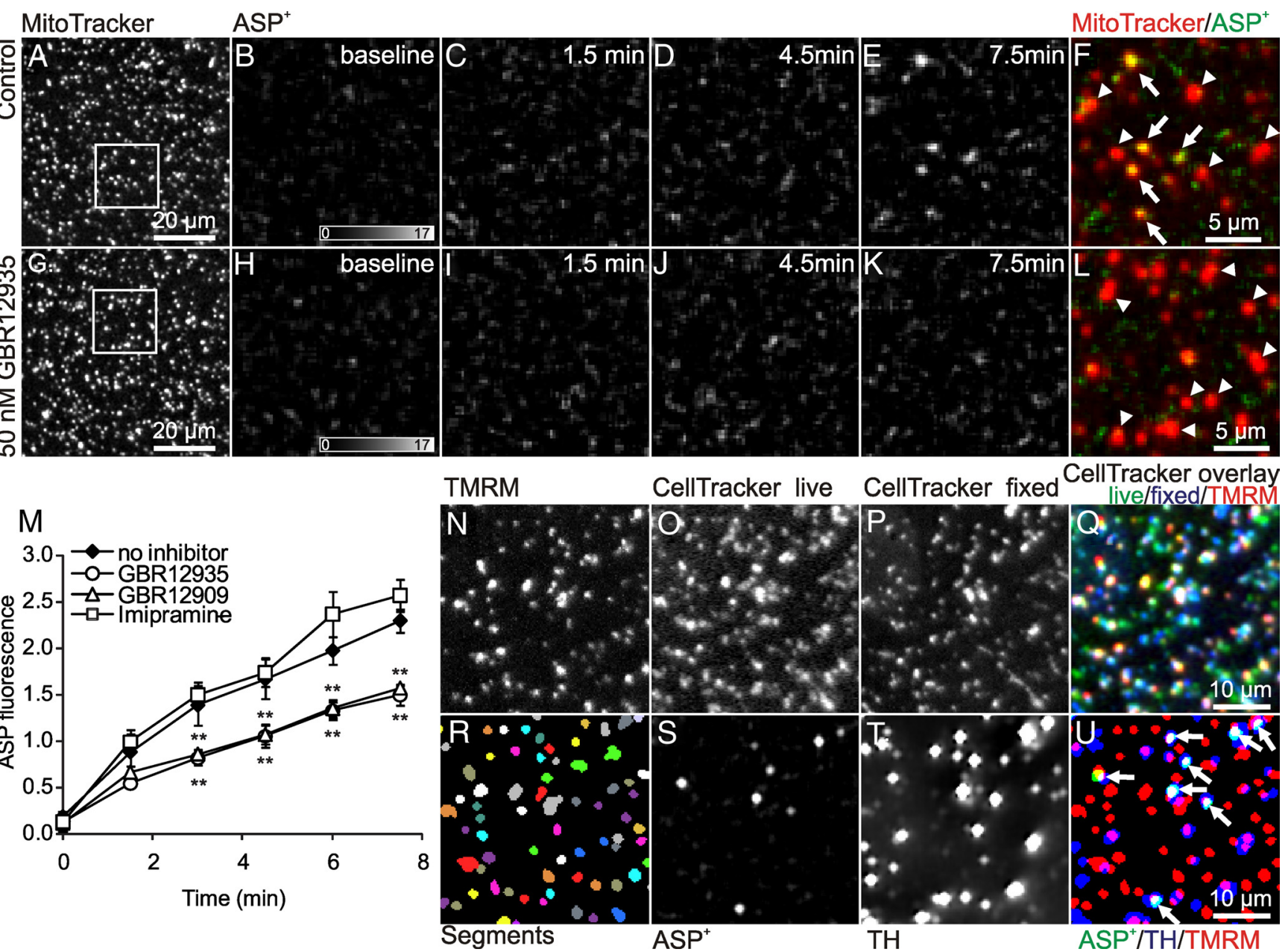

Figure 4. $\mathrm{ASP}^{+}$accumulates in synaptosomes specifically via the dopamine transporter and colocalizes with $\mathrm{TH} . \boldsymbol{A}-\boldsymbol{M}$, Nonpurified synaptosomes were stained by MitoTracker Red $(\boldsymbol{A}, \boldsymbol{G})$ and the

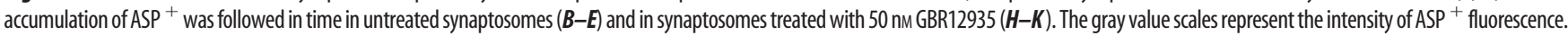
$\boldsymbol{F}, \boldsymbol{L}$, Composite image for the last time point. The arrows and arrowheads indicate ASP ${ }^{+}$and MitoTracker-positive and MitoTracker-only objects, respectively. Images shown were high-pass and Wiener filtered and scaled identically.M,ASP ${ }^{+}$fluorescence intensities were measured over MitoTracker Red-positive pixels after baseline subtraction. The effects of GBR12935 (50 nm; circles), GBR12909 (10 nm; triangles), and imipramine (100 nм; squares) on ASP ${ }^{+}$accumulation in synaptosomes were monitored. Data indicate means \pm SE $\left(n=4\right.$, each the average of 3 technical replicates); ${ }^{* *} p<0.01$ compared with untreated control (diamonds) at the given time point. $\boldsymbol{N}-\boldsymbol{U}$, Colocalization of the vital probe ASP ${ }^{+}$with immunofluorescence after fixation. $\boldsymbol{N}-\boldsymbol{P}$, The patterns of TMRM and CellTracker were used to align images taken before and after fixation. The composite image $(\boldsymbol{Q})$ indicates a good alignment. The TMRM image $(\boldsymbol{N})$ was segmented $(\boldsymbol{R})$, and these objects (marked by different colors) were used to determine the presence or absence of $\mathrm{ASP}^{+}(\boldsymbol{S})$ and $\mathrm{TH}(\boldsymbol{T})$ in the corresponding binarized images $(\boldsymbol{U})$ where arrows indicate ASP ${ }^{+}$colocalization with TH. $\boldsymbol{S}, \boldsymbol{I}$, Images were high-pass and Savitzky-Golay smooth filtered.

Table 4. Concentrations for half-maximum inhibition $\left(K_{\mathrm{i}}\right)$ of three inhibitors

\begin{tabular}{lcl}
\hline & $K_{\mathrm{i}}$ for dopamine transporter & $K_{\mathrm{i}}$ for other transporters \\
\hline Imipramine & $7.5 \mu \mathrm{M}$ & Serotonin $(7.7 \mathrm{~nm})$, norepinephrine $(67 \mathrm{~nm})$ \\
GBR12935 & $21.5 \mathrm{nM}$ & Serotonin $(6.5 \mu \mathrm{M})$, norepinephrine $(225 \mathrm{~nm})$ \\
GBR12909 & $1 \mathrm{nM}$ & $100 \mathrm{~nm}$ or more \\
\hline
\end{tabular}

Values are from Anderson (1989) and Torres et al. (2003). ever, only $22 \pm 2 \%$ of tyrosine hydroxylase-positive objects were also $\mathrm{ASP}^{+}$-positive. This population of TH-positive and $\mathrm{ASP}^{+}$-negative synaptosomes is likely explained by the different distribution pattern of $\mathrm{TH}$ and dopamine transporters in dopaminergic axons in the striatum. Although TH is present throughout the axoplasm and in synaptic varicosities (Freund et al., 1984; Nirenberg et al., 1996), the dopamine transporter is enriched in varicose segments of dopaminergic axons ( $\mathrm{Ni}$ - 


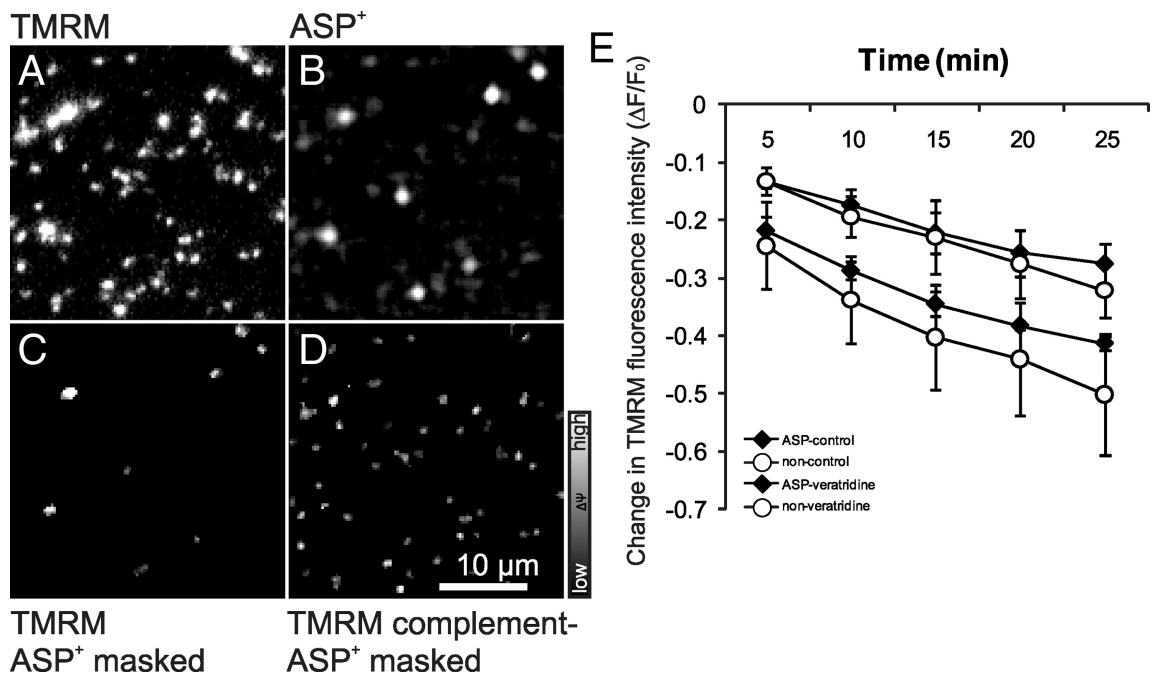

Figure 5. Stress responses of TMRM fluorescence in dopaminergic and nondopaminergic striatal synaptosomes from young mice. Nonfractionated striatal synaptosomes were stained with $\operatorname{TMRM}(\boldsymbol{A})$ and after the functional time lapse assays with ASP $^{+}$ $(\boldsymbol{B})$. TMRM fluorescence of dopaminergic synaptosomes was measured over the intersection of shapes determined by maximal intensity projected TMRM time lapse and ASP ${ }^{+}$fluorescence $(\boldsymbol{C})$; that of nondopaminergic synaptosomes was measured over the complement set $(\boldsymbol{D})$. $\boldsymbol{E}$, TMRM fluorescence intensity changes evoked by veratridine (1 $\mu \mathrm{M})$ were monitored for $25 \mathrm{~min}$. Data indicate means \pm SE ( $n=3$ biological replicates, each the average of 3 technical replicates).

renberg et al., 1996; Hersch et al., 1997). Thus, these THpositive and $\mathrm{ASP}^{+}$-negative objects may not be bona fide synaptosomes, but intervaricose segments of dopaminergic axons.

These results indicate that $\mathrm{ASP}^{+}$association with synaptosomes occurs on the dopamine transporter and that the $7 \%$ of synaptosomes derived from presynaptic varicosities in dopaminergic neuronal axons in mixed synaptosomes prepared by classical Percoll extraction can be selectively distinguished with fluorescence microscopy and image processing. $\mathrm{ASP}^{+}$ labeling may be a better discriminator than $\mathrm{TH}$ labeling since it does not pick up objects derived from intervaricose segments of dopaminergic axons. However, if bioenergetics in the wider dopaminergic neuronal cell is of interest, postimmunofluorescence $\mathrm{TH}$ tagging can be used to classify this population in live fluorescence microscopy experiments as described here.

The bioenergetic responses of striatal dopaminergic and nondopaminergic synaptosomes from young and old mice to applied stresses

To compare the bioenergetic competence of dopaminergic and nondopaminergic synaptosomes after various stresses, we recorded TMRM fluorescence, an index of changes in the sum of plasma membrane and mitochondrial membrane potentials (Nicholls, 2006). Dopaminergic and nondopaminergic synaptosomes were distinguished using both $\mathrm{ASP}^{+}$tagging (Fig. 5A-D) and $\mathrm{TH}$ tagging (data not shown) at the end of each experiment. After recording baseline TMRM fluorescence, a set of treatments was applied (Table 5), and the treatment-evoked membrane potential depolarization was calculated in millivolts. Synaptosomes were challenged bioenergetically by (1) disturbance in ion homeostasis (high extracellular $\left[\mathrm{K}^{+}\right] /\left[\mathrm{Ca}^{2+}\right]$ to depolarize the plasma membrane, open voltage-gated $\mathrm{Ca}$ channels, and raise cytoplasmic $\left[\mathrm{Ca}^{2+}\right]$ or veratridine/ouabain/high extracellular $\left[\mathrm{Ca}^{2+}\right]$ to activate $\mathrm{Na}$ channels, inhibit $\mathrm{Na}$ export, and drive $\mathrm{Na} / \mathrm{Ca}$ exchanges to raise cytoplasmic $\left.\left[\mathrm{Ca}^{2+}\right]\right)$; (2) inhibition of electron transport (rotenone to inhibit complex I or 3-nitropropionic acid to inhibit complex II); (3) insult by reactive oxygen species (superoxide production by xanthine/xanthine oxidase, $t$-butyl hydroperoxide, or $\mathrm{H}_{2} \mathrm{O}_{2}$ ), or a combination of these. The dose-response for each treatment was determined using unseparated cortical synaptosomes from young mice (data not shown). The minimum concentration that provided a significant depolarization when compared with no treatment was chosen for experiments, to prevent massive signal loss caused by synaptosomal death. All insults tested decreased the combined membrane potential. Figure $5 E$ shows the TMRM fluorescence change with a representative treatment (veratridine). Table 5 shows that decreases in membrane potential under stress did not differ significantly between the dopaminergic and $\mathrm{ASP}^{+}$-negative nondopaminergic synaptosomal subpopulations in the same microscope field.

The responses described above were measured in synaptosomes derived from both young and old mice to determine whether the dopaminergic synaptosomes acquired greater sensitivity to stress in aged brain. No differences were found in the relative amounts of dopaminergic synaptosomes $\left(\mathrm{ASP}^{+}\right.$- or $\mathrm{TH}-$ positive) between young and old mice (data not shown). The changes in membrane potential under various stresses were not significantly different between young and old mice using either $\mathrm{ASP}^{+}$tagging (Table 5) or TH tagging (data not shown).

\section{Discussion}

Our results show that dopaminergic synaptosomes separated using immunomagnetic beads respired more slowly per microgram of protein than those in the nondopaminergic flow-through fraction. However, detailed analysis revealed that this difference was attributable to fewer respiration-competent synaptosomes per microgram of protein in the separated dopaminergic fraction; respiration rates per $10^{6}$ respiration-competent synaptosomes were the same in the two fractions. In agreement with this result, the bioenergetic responses to stress of $\mathrm{ASP}^{+}$-tagged dopaminergic synaptosomes in unseparated populations did not differ from those of $\mathrm{ASP}^{+}$-negative synaptosomes in the same microscope field.

We found that dopaminergic synaptosomes from aged mice maintained membrane potentials as effectively as those from young mice. Whether aging affects mitochondrial energy metabolism is still debated (Evans et al., 1998; Joyce et al., 2003; Nicholls, 2004; Parihar and Brewer, 2007; Kilbride et al., 2008); however, others have reported results compatible with ours (Solmi et al., 1994; Evans et al., 1998; Joyce et al., 2003; Kilbride et al., 2008). For example, Kilbride et al. (2008) found similar decreases in membrane potential in unfractionated brain synaptosomes from young and aged rats in response to inhibition of energy metabolism by rotenone, oligomycin, antimycin A, or FCCP (although basal membrane potentials were lower in old animals). Evans et al. (1998) reported that resting plasma membrane potentials from neurons isolated from old rats were similar to those of young rats. Another study using rat whole-brain synaptosomes showed no difference in resting oxygen consumption rate, mitochondrial and plasma membrane potential, maximal 
Table 5. Dopaminergic ASP ${ }^{+}$-positive synaptosomes maintain TMRM fluorescence as effectively as ASP ${ }^{+}$-negative nondopaminergic synaptosomes under various stresses and this capacity does not decline with age

\begin{tabular}{|c|c|c|c|c|c|c|}
\hline \multirow[b]{3}{*}{ Stimuli } & \multirow[b]{3}{*}{ Working concentration } & \multirow[b]{3}{*}{ Type of stress } & \multicolumn{4}{|c|}{ Membrane potential } \\
\hline & & & \multicolumn{2}{|l|}{ Young } & \multicolumn{2}{|l|}{ Old } \\
\hline & & & $\mathrm{ASP}^{+}$-positive & $\mathrm{ASP}^{+}$-negative & $\mathrm{ASP}^{+}$-positive & $\mathrm{ASP}^{+}$-negative \\
\hline t-Butyl hydroperoxide & $1 \mathrm{~mm}$ & Lipid peroxidation & $-21.7 \pm 6.1$ & $-25.1 \pm 4.2$ & $-19.4 \pm 14.4$ & $-25.1 \pm 6.4$ \\
\hline Veratridine & $1 \mu \mathrm{M}$ & $\mathrm{Na} / \mathrm{Ca}$ influx & $-14.5 \pm 0.6$ & $-18.5 \pm 2.1$ & $-20.1 \pm 5.7$ & $-22.2 \pm 4.8$ \\
\hline $\mathrm{H}_{2} \mathrm{O}_{2}$ & $200 \mu \mathrm{m}$ & ROS & $-14.1 \pm 3.9$ & $-15.7 \pm 2.9$ & $-12.6 \pm 2.1$ & $-15.4 \pm 2.2$ \\
\hline Veratridine + ouabain $+[\mathrm{Ca}]_{\mathrm{ex}}$ & $1 \mu \mathrm{m} / 100 \mu \mathrm{M} / 10 \mathrm{~mm}$ & Ca influx & $-22.5 \pm 1.2$ & $-25.6 \pm 1.9$ & $-31.9 \pm 6.9$ & $-25.6 \pm 1.7$ \\
\hline$[\mathrm{K}]_{\mathrm{ex}}+[\mathrm{Ca}]_{\mathrm{ex}}$ & $30 \mathrm{~mm} / 10 \mathrm{~mm}$ & Ca influx & $-33.6 \pm 1.1$ & $-36.9 \pm 2.8$ & $28.4 \pm 3.1$ & $-35.8 \pm 1.0$ \\
\hline Veratridine $+\mathrm{H}_{2} \mathrm{O}_{2}$ & $1 \mu \mathrm{m} / 200 \mu \mathrm{M}$ & $\mathrm{Na} / \mathrm{Ca}$ influx $+\mathrm{ROS}$ & $-20.3 \pm 11.4$ & $-21.2 \pm 6.9$ & $-20.5 \pm 6.6$ & $-22.7 \pm 5.3$ \\
\hline Rotenone $+\mathrm{H}_{2} \mathrm{O}_{2}$ & $10 \mathrm{~nm} / 200 \mu \mathrm{M}$ & ETC inhibition + ROS & $-16.6 \pm 6.2$ & $-22.3 \pm 6.2$ & $19.2 \pm 2.6$ & $-21.1 \pm 2.6$ \\
\hline $3 \mathrm{NP}+\mathrm{H}_{2} \mathrm{O}_{2}$ & $400 \mu \mathrm{M} / 200 \mu \mathrm{M}$ & ETC inhibition + ROS & $-18.2 \pm 9.2$ & $-21.8 \pm 5.7$ & $11.4 \pm 3.4$ & $-18.0+6.6$ \\
\hline
\end{tabular}

Striatal synaptosomes were prepared from young ( 5 month old) or old ( 28 month old) mice of either gender and the decrease in TMRM fluorescence intensity in ASP ${ }^{+}$-positive and ASP ${ }^{+}$-negative individual synaptosomes caused by various drug treatments for $25 \mathrm{~min}$ was determined and calculated as the decrease in plasma membrane plus mitochondrial membrane potential. Data indicate means \pm SE ( $n=3$ biological replicates, each the average of 3 technical replicates). No differences were significant (one-way ANOVA on dopaminergic vs nondopaminergic synaptosomes and young vs old mice).

ETC, Electron transport chain; ROS, reactive oxygen species.

phosphorylation capacity, and proton leak, but lower respiration was observed in the presence of uncoupler when whole-brain synaptosomes from aged rat were compared with those from young rat (Joyce et al., 2003).

Our results do not support studies indicating impaired mitochondrial function in dopamine-enriched neurons (Rabinovic et al., 2000; Cantuti-Castelvetri et al., 2003; Dawson and Dawson, 2003; Brenner-Lavie et al., 2008, 2009; Miyazaki and Asanuma, 2008; Bisaglia et al., 2010) and/or that aging exaggerates these impairments (Bertoni-Freddari et al., 1993, 1996, 2006; Bowling et al., 1993; Ferrándiz et al., 1994; Guerrieri et al., 1996; Antier et al., 2004). There are possible explanations of this discrepancy. First, Davey et al. (1998) reported that there is a "threshold effect" of the inhibition of complex I. Mitochondria in dopaminergic neurons may function normally because a decrease in complex I activity is too small to affect function. Second, dopaminergic neurons may have less mitochondrial capacity, but not in presynaptic varicosities or intervaricose axonal regions. Liang et al. (2007) reported that dopaminergic dendrites in mouse brain sections have lower mitochondrial volume fractions than nondopaminergic dendrites, implying weaker mitochondrial capacity in postsynaptic dopaminergic terminals rather than in the presynaptic synaptosomes investigated here. Third, fluorescent probes of membrane potential must be interpreted very carefully; several studies wrongly report decreases in membrane potential as a function of aging because of incorrect interpretation of the fluorescent signal (Nicholls, 2004). Finally, loss of synaptic terminals is well known in the aging brain (Bertoni-Freddari et al., 1996; Peters et al., 2008) or in brain pathogenesis (Scheff et al., 1990; Betarbet et al., 2000). However, loss of function appears to trigger a compensatory reaction. Several studies report that mitochondria in old animals decrease in number but increase in size, maintaining the mitochondrial volume fraction (Bertoni-Freddari et al., 1993, 2006; Solmi et al., 1994). This phenomenon, together with synaptic enlargement during aging (Bertoni-Freddari et al., 1992), may support our finding of no bioenergetic defects in synaptic terminals from aging striatum.

The lower number of respiration-competent synaptosomes in the immunomagnetically purified dopaminergic fraction may have roots in the underlying anatomy. In contrast to typical glutamatergic synaptic end terminals, dopaminergic presynaptic terminals form predominantly en passant boutons (Arluison et al., 1984; Williams and Goldman-Rakic, 1993). These boutons may genuinely contain less mitochondria, or mitochondria may be only transiently present near synapses while traveling on microtubule tracks passing through the bouton. This anatomical difference may affect synaptosome preparation, as axons severed during the homogenization step have to close at two ends around en passant boutons compared with end terminals.

Immunomagnetic bead separation should enrich the quantity of synaptosomes per microgram of protein. Higher nonfunctional protein in dopaminergic synaptosome fractions may reflect the underlying biology, but possible preparation artifacts include different amounts of "invisible" free mitochondria and the presence of bound antibody causing overestimation of synaptosomal protein. In contrast, in unfractionated striatal preparations, the percentage of $\mathrm{TH}$-stained synaptosomes that were also TMRM-positive tended to be larger than that of TH-negative synaptosomes, emphasizing the concern that the lower number of respiration-competent synaptosomes in the dopaminergic fraction may result from the separation technology.

An important aspect of this work was to improve techniques to assess the bioenergetic status of mitochondria in synaptosomes isolated from different cell types. Although the synaptosome preparation has been used for almost 50 years and in $>12,000$ publications, the inherent heterogeneity of the preparation has never before been fully addressed. Studies from the groups of Nichols (Nichols and Mollard, 1996) and Sánchez-Prieto (Millán et al., 2002) have allowed the transmitter subtype of single synaptosomes to be identified among aggregates, but this has not previously been performed on entire microscopic fields of synaptosomes or combined with a bioenergetic analysis with criteria to exclude nonfunctional organelles. We previously introduced a method to generate a monolayer of attached single synaptosomes, combined with algorithms to analyze the response from several hundred synaptosomes in a field-of-view individually and simultaneously with algorithmic image processing to improve objectivity and accuracy in data analysis (Choi et al., 2009). In the present study, we further refine these techniques to include fluorescence methods to determine the mitochondrial volume fraction, and to assess the bioenergetic status of individual synaptosomes and determine which were respiration-competent and, by $\mathrm{ASP}^{+}$transport, possessed the DA transporter.

These techniques have several advantages over electron microscopy approaches. First, fixation is not required for sample 
preparation, eliminating issues of sample shrinkage. Second, the procedures are simpler, quicker, and more economical. Third, in situ live mitochondrial morphology can be measured. Finally, the assays enable the selection of bioenergetic or respirationcompetent synaptosomes for data analysis, by excluding extraneous objects such as broken synaptosomes or nonsynaptic material. This last advantage is particularly important in experiments using synaptosomes because of their unique heterogeneity. Joyce et al. (2003) used electron microscopy to quantify whole rat brain synaptosomes and found that $\sim 68 \%$ of the structures in a synaptosome preparation contained synaptic vesicles and, of these, only $38 \%$ contained mitochondria. In our study using mouse brain regions and a different synaptosomal preparation method, $\sim 70 \%$ of objects were viable synaptosomes. In addition, the preparation also contains broken membranes visible by electron microscopy, but invisible to our entrapped fluorescence probe method. Therefore, $>30 \%$ of protein in prepared synaptosomes does not contribute to the measured functions. It is critical to exclude the values from these contaminants to obtain accurate measurements from the synaptosomal population. However, microscopy of unfractionated monolayers has an inherent drawback: unlike the separated synaptosomal fractions in Figure 1, identification of dopaminergic synaptosomes in mixed monolayers does not allow the respiration rates of different subpopulations to be distinguished using conventional respirometry.

In summary, our results do not support the hypotheses that mitochondrial bioenergetic capacity is intrinsically lower in striatal dopaminergic synaptosomes, making them unusually susceptible to inhibition of electron transport by oxidative damage, or that this property is accentuated by age and underlies susceptibility to Parkinson's disease.

\section{References}

Andersen PH (1989) The dopamine inhibitor GBR 12909: selectivity and molecular mechanism of action. Eur J Pharmacol 166:493-504.

Antier D, Carswell HV, Brosnan MJ, Hamilton CA, Macrae IM, Groves S, Jardine E, Reid JL, Dominiczak AE (2004) Increased levels of superoxide in brains from old female rats. Free Radic Res 38:177-183.

Arluison M, Dietl M, Thibault J (1984) Ultrastructural morphology of dopaminergic nerve terminals and synapses in the striatum of the rat using tyrosine hydroxylase immunocytochemistry: a topographical study. Brain Res Bull 13:269-285.

Bertoni-Freddari C, Fattoretti P, Pieroni M, Meier-Ruge W, Ulrich J (1992) Enlargement of synaptic size as a compensative reaction in aging and dementia. Pathol Res Pract 188:612-615.

Bertoni-Freddari C, Fattoretti P, Casoli T, Spagna C, Meier-Ruge W, Ulrich J (1993) Morphological plasticity of synaptic mitochondria during aging. Brain Res 628:193-200.

Bertoni-Freddari C, Fattoretti P, Paoloni R, Caselli U, Galeazzi L, Meier-Ruge W (1996) Synaptic structural dynamics and aging. Gerontology 42:170-180.

Bertoni-Freddari C, Mocchegiani E, Malavolta M, Casoli T, Di Stefano G, Fattoretti P (2006) Synaptic and mitochondrial physiopathologic changes in the aging nervous system and the role of zinc ion homeostasis. Mech Ageing Dev 127:590-596.

Betarbet R, Sherer TB, MacKenzie G, Garcia-Osuna M, Panov AV, Greenamyre JT (2000) Chronic systemic pesticide exposure reproduces features of Parkinson's disease. Nat Neurosci 3:1301-1306.

Bisaglia M, Soriano ME, Arduini I, Mammi S, Bubacco L (2010) Molecular characterization of dopamine-derived quinones reactivity toward NADH and glutathione: implications for mitochondrial dysfunction in Parkinson disease. Biochim Biophys Acta 1802:699-706.

Bowling AC, Mutisya EM, Walker LC, Price DL, Cork LC, Beal MF (1993) Age-dependent impairment of mitochondrial function in primate brain. J Neurochem 60:1964-1967.

Brenner-Lavie H, Klein E, Zuk R, Gazawi H, Ljubuncic P, Ben-Shachar D (2008) Dopamine modulates mitochondrial function in viable SH-SY5Y cells possibly via its interaction with complex I: relevance to dopamine pathology in schizophrenia. Biochim Biophys Acta 1777:173-185.

Brenner-Lavie H, Klein E, Ben-Shachar D (2009) Mitochondrial complex I as a novel target for intraneuronal DA: modulation of respiration in intact cells. Biochem Pharmacol 78:85-95.

Calabresi P, Centonze D, Bernardi G (2000) Cellular factors controlling neuronal vulnerability in the brain: a lesson from the striatum. Neurology 55:1249-1255.

Cantuti-Castelvetri I, Shukitt-Hale B, Joseph JA (2003) Dopamine neurotoxicity: age-dependent behavioral and histological effects. Neurobiol Aging 24:697-706.

Chinta SJ, Kumar MJ, Hsu M, Rajagopalan S, Kaur D, Rane A, Nicholls DG, Choi J, Andersen JK (2007) Inducible alterations of glutathione levels in adult dopaminergic midbrain neurons result in nigrostriatal degeneration. J Neurosci 27:13997-14006.

Choi SW, Gerencser AA, Nicholls DG (2009) Bioenergetic analysis of isolated cerebrocortical nerve terminals on a microgram scale: spare respiratory capacity and stochastic mitochondrial failure. J Neurochem 109:1179-1191.

Davey GP, Canevari L, Clark JB (1997) Threshold effects in synaptosomal and nonsynaptic mitochondria from hippocampal CA1 and paramedian neocortex brain regions. J Neurochem 69:2564-2570.

Davey GP, Peuchen S, Clark JB (1998) Energy thresholds in brain mitochondria. Potential involvement in neurodegeneration. J Biol Chem 273:12753-12757.

Dawson TM, Dawson VL (2003) Molecular pathways of neurodegeneration in Parkinson's disease. Science 302:819-822.

Dunkley PR, Jarvie PE, Robinson PJ (2008) A rapid Percoll gradient procedure for preparation of synaptosomes. Nat Protoc 3:1718-1728.

Evans MS, Collings MA, Brewer GJ (1998) Electrophysiology of embryonic, adult and aged rat hippocampal neurons in serum-free culture. J Neurosci Methods 79:37-46.

Ferrándiz ML, Martínez M, De Juan E, Díez A, Bustos G, Miquel J (1994) Impairment of mitochondrial oxidative phosphorylation in the brain of aged mice. Brain Res 644:335-338.

Freund TF, Powell JF, Smith AD (1984) Tyrosine hydroxylase-immunoreactive boutons in synaptic contact with identified striatonigral neurons, with particular reference to dendritic spines. Neuroscience 13:1189-1215.

Gerencser AA, Adam-Vizi V (2001) Selective, high-resolution fluorescence imaging of mitochondrial $\mathrm{Ca}^{2+}$ concentration. Cell Calcium 30:311-321.

Gerencser AA, Nicholls DG (2008) Measurement of instantaneous velocity vectors of organelle transport: mitochondrial transport and bioenergetics in hippocampal neurons. Biophys J 95:3079-3099.

Gerencser AA, Doczi J, Töröcsik B, Bossy-Wetzel E, Adam-Vizi V (2008) Mitochondrial swelling measurement in situ by optimized spatial filtering: astrocyte-neuron differences. Biophys J 95:2583-2598.

Gerencser AA, Neilson A, Choi SW, Edman U, Yadava N, Oh RJ, Ferrick DA, Nicholls DG, Brand MD (2009) Quantitative microplate-based respirometry with correction for oxygen diffusion. Anal Chem 81:6868-6878.

Guerrieri F, Vendemiale G, Turturro N, Fratello A, Furio A, Muolo L, Grattagliano I, Papa S (1996) Alteration of mitochondrial $\mathrm{F}_{0} \mathrm{~F}_{1}$ ATP synthase during aging. Possible involvement of oxygen free radicals. Ann N Y Acad Sci 786:62-71.

Hersch SM, Yi H, Heilman CJ, Edwards RH, Levey AI (1997) Subcellular localization and molecular topology of the dopamine transporter in the striatum and substantia nigra. J Comp Neurol 388:211-227.

Joyce OJ, Farmer MK, Tipton KF, Porter RK (2003) Oxidative phosphorylation by in situ synaptosomal mitochondria from whole brain of young and old rats. J Neurochem 86:1032-1041.

Kilbride SM, Telford JE, Davey GP (2008) Age-related changes in $\mathrm{H}_{2} \mathrm{O}_{2}$ production and bioenergetics in rat brain synaptosomes. Biochim Biophys Acta 1777:783-788.

Kumar MJ, Nicholls DG, Andersen JK (2003) Oxidative alpha-ketoglutarate dehydrogenase inhibition via subtle elevations in monoamine oxidase $\mathrm{B}$ levels results in loss of spare respiratory capacity: implications for Parkinson's disease. J Biol Chem 278:46432-46439.

Liang CL, Wang TT, Luby-Phelps K, German DC (2007) Mitochondria mass is low in mouse substantia nigra dopamine neurons: implications for Parkinson's disease. Exp Neurol 203:370-380.

Mason JN, Farmer H, Tomlinson ID, Schwartz JW, Savchenko V, DeFelice LJ, Rosenthal SJ, Blakely RD (2005) Novel fluorescence-based approaches 
for the study of biogenic amine transporter localization, activity, and regulation. J Neurosci Methods 143:3-25.

Millán C, Luján R, Shigemoto R, Sánchez-Prieto J (2002) Subtype-specific expression of group III metabotropic glutamate receptors and $\mathrm{Ca}^{2+}$ receptors in single nerve terminals. J Biol Chem 277:47796-47803.

Miyazaki I, Asanuma M (2008) Dopaminergic neuron-specific oxidative stress caused by dopamine itself. Acta Med Okayama 62:141-150.

Mizuno Y, Ohta S, Tanaka M, Takamiya S, Suzuki K, Sato T, Oya H, Ozawa T, Kagawa Y (1989) Deficiencies in complex I subunits of the respiratory chain in Parkinson's disease. Biochem Biophys Res Commun 163:1450-1455.

Nicholls DG (2004) Mitochondrial membrane potential and aging. Aging Cell 3:35-40.

Nicholls DG (2006) Simultaneous monitoring of ionophore- and inhibitormediated plasma and mitochondrial membrane potential changes in cultured neurons. J Biol Chem 281:14864-14874.

Nichols RA, Mollard P (1996) Direct observation of serotonin 5- $\mathrm{HT}_{3}$ receptor-induced increases in calcium levels in individual brain nerve terminals. J Neurochem 67:581-592.

Nirenberg MJ, Vaughan RA, Uhl GR, Kuhar MJ, Pickel VM (1996) The dopamine transporter is localized to dendritic and axonal plasma membranes of nigrostriatal dopaminergic neurons. J Neurosci 16:436-447.

Parihar MS, Brewer GJ (2007) Simultaneous age-related depolarization of mitochondrial membrane potential and increased mitochondrial reactive oxygen species production correlate with age-related glutamate excitotoxicity in rat hippocampal neurons. J Neurosci Res 85:1018-1032.

Peters A, Sethares C, Luebke JI (2008) Synapses are lost during aging in the primate prefrontal cortex. Neuroscience 152:970-981.

Rabinovic AD, Lewis DA, Hastings TG (2000) Role of oxidative changes in the degeneration of dopamine terminals after injection of neurotoxic levels of dopamine. Neuroscience 101:67-76.

Richardson JR, Caudle WM, Guillot TS, Watson JL, Nakamaru-Ogiso E, Seo BB, Sherer TB, Greenamyre JT, Yagi T, Matsuno-Yagi A, Miller GW (2007) Obligatory role for complex I inhibition in the dopaminergic neurotoxicity of 1-methyl-4-phenyl-1,2,3,6-tetrahydropyridine (MPTP). Toxicol Sci 95:196-204.

Schapira AH, Cooper JM, Dexter D, Jenner P, Clark JB, Marsden CD (1989) Mitochondrial complex I deficiency in Parkinson's disease. Lancet 1:1269.

Scheff SW, DeKosky ST, Price DA (1990) Quantitative assessment of cortical synaptic density in Alzheimer's disease. Neurobiol Aging 11:29-37.

Shoffner JM, Watts RL, Juncos JL, Torroni A, Wallace DC (1991) Mitochondrial oxidative phosphorylation defects in Parkinson's disease. Ann Neurol 30:332-339.

Solmi R, Pallotti F, Rugolo M, Genova ML, Estornell E, Ghetti P, Pugnaloni A, Biagini G, Rizzoli C, Lenaz G (1994) Lack of major mitochondrial bioenergetic changes in cultured skin fibroblasts from aged individuals. Biochem Mol Biol Int 33:477-484.

Torres GE, Gainetdinov RR, Caron MG (2003) Plasma membrane monoamine transporters: structure, regulation and function. Nat Rev Neurosci 4:13-25.

Williams SM, Goldman-Rakic PS (1993) Characterization of the dopaminergic innervation of the primate frontal cortex using a dopaminespecific antibody. Cereb Cortex 3:199-222.

Xiang JZ, Morton J, Brammer MJ, Campbell IC (1990) Regulation of calcium concentrations in synaptosomes: alpha 2-adrenoceptors reduce free $\mathrm{Ca}^{2+}$ by closure of N-type $\mathrm{Ca}^{2+}$ channels. J Neurochem 55:303-310. 